\title{
SARS-CoV-2 Triggers Inflammatory and Cell Death in Experimental Infection by Activating Human Cells Caspases
}

\author{
Jardel Fábio Lopes Ferreira ${ }^{1}$, Murilo Tavares Amorim ${ }^{1 *}$, \\ Francisco Canindé Ferreira de Luna ${ }^{1}$, Gustavo Moraes Holanda ${ }^{2}$, \\ Clebson Pantoja Pimentel ${ }^{2}$, Walter Félix Franco $\mathrm{Neto}^{3}$, Caroline Carvalho Pinto ${ }^{3}$, \\ Ana Paula Sousa Araújo ${ }^{3}$, Gleissy Adriane Lima Borges ${ }^{3}$ and Ana Cecília Ribeiro Cruz ${ }^{1}$ \\ ${ }^{1}$ Institute of Biological Sciences, Federal University of Pará, S. Augusto Corrêa, 01 - Guamá, \\ Belém - PA, 66075-110, Brazil \\ ${ }^{2}$ University of the Amazon, S. Quintino Bocaiúva, 1808 - Nazaré, \\ Belém - PA, 66035-190, Brazil \\ ${ }^{3}$ Arbovirology and Hemorrhagic Fevers Section, Evandro Chagas Institute, Highway BR-316 \\ $\mathrm{km} 7 \mathrm{~s} / \mathrm{n}$ - Levilândia, PA, 67030-000, Brazil \\ *Corresponding author
}

Keywords

Cell shrinkage, macrophages, parenchyma, phagocytic degradation

Article Info

Accepted:

25 March 2021

Available Online:

10 April 2021
Apoptosis is a process that occurs in programmed physiological and nonphysiological cell death. In morphological aspect, among apoptosis products, cell shrinkage, subsequent to the degeneration of the nucleus characterized by condensation of chromatin, followed by the nuclear membrane disappearance. In particular, apoptosis modulation in SARS-CoV infection seems to be directly associated with specific cell-type interactions, which can significantly influence the manifestations resulting from the pathology. Therefore, it is noted the need for studies that promote debates about the modulation of apoptosis in animals and humans, infected by SARS-CoV. Because the spread of SARS-CoV-2 has become the most recent threat to global health, the quest to understand the virus-host interaction mechanisms has become a challenge for public health, research and medical communities, especially due to the interposition of factors related to the evolutionary spectrum of the agent. Thus, given the urgency of the global health situation, the need to develop therapies and vaccines to combat Covid-19 due to the SARS-CoV-2 pandemic that the recent experimental data on the main molecules expressed during infection by SARS-CoV-2, can be an alternative for the development of new effective parameters in the control of the disease, is the recognition and the differential parallelism between the mediating molecules, by which the immune system recognizes and responds to specific $\mathrm{CoV}$ infections, acting to support the development of therapeutic strategies. 


\section{Introduction}

Apoptosis is a process that occurs in programmed physiological and nonphysiological cell death. In morphological aspect, among apoptosis products, cell shrinkage, subsequent to the degeneration of the nucleus characterized by condensation of chromatin, followed by the nuclear membrane disappearance (Lavrik; Golks; Krammer, 2005; Ziegler; Groscurth, 2004). The membrane vesicle is followed continuously by these conditions, thus causing the breakdown of the nucleus (Cariorrexis). This causes the cells to detach from their surrounding tissues and to separate based on cellular fragments, from the cytoplasm embedded in compacted organelles. Subsequently, apoptotic bodies have the function of assuming the sprouting process. At the end of this process, cell surface markers (Phosphatidylserine) are released from the plasma membrane, in order to attract some cells, especially macrophages and parenchyma, acting to phagocytize apoptotic bodies and prevent secondary necrosis. In general, it is known that apoptosis is not a process resulting from a single condition, but rather a specific set of conditions, previously established, that corroborate the action of regulatory mechanisms, which trigger active responses conditioned to cell death (Savill; Fadok, 2000; Ziegler; Groscurth, 2004).

Therefore, cells in apoptosis process, trigger the activation of numerous proteins, in a regulated sequence both temporally and spatially. Its initiation occurs from the induction of a series of stimuli, conventionally included in the connection of ligands to the receptors of the cell surface of the Tumor Necrosis Factor (TNF). Some conditions mentioned above, such as the cleavage of products originating from proteins, DNA and phagocytic degradation, are considered associated final products, through several biochemical processes and their final execution is caspase dependent (Kurosaka et al., 2003; Zeiss, 2003; Ziegler; Groscurth, 2004).

Caspases are cysteine proteases divided into pro-caspases, capable of initiating the apoptotic process and effector caspases that are responsible for the cleavage of aspartic residue after its activation. Thus, the proenzymes assist other pro-caspases activation, in order to initiate the protein cascade and perform various cell death processes (Zeiss, 2003). The necessary stimuli for apoptotic factors activation consist of the release of mediators that will result in cell death, in order to mediate cell proliferation and control cell development and homeostasis maintenance. Therefore, this phenomenon can be triggered by intrinsic and extrinsic pathways, especially the conditions associated with viral infections (Kurosaka et al., 2003; Obeng, 2021).

It is believed that the apoptosis process can be modulated by some viruses, being related to the coding of their viral replication products. The induction or inhibition of apoptosis in infected cells, has been widely discussed, as being one of the most important questions related to viral pathogenesis (Obeng, 2021; Zhang, et al., 2007).

The extrinsic pathway of apoptosis occurs through the interaction of death receptors (DRs dead receptors), with their respective ligands. These DRs, belong to the family TNFR (tumor necrosis factor receptor), having FAZ, TNF-R1 and TRAIL-R1 (TNF-related apoptosis-inducing ligand receptor 1 and 2) and their respective FASL ligands (FASligand), TNF- $\alpha$ and TRAIL. In particular, FAS (CD95/APO-1) stands out as the prototype of death receptors, being expressed constitutively in most cell tissues, characterized mainly by the presence of up to six cysteine-rich domains in its extracellular portions, responsible for the connection with FASL 
(CD95L / APO-1L). This link between trimers of FAS and FASL, results in the recruitment of adapter proteins called FADD (FASassociated death domain), through the interaction between the DD domains (death domain) present in both this molecule and in the FAS receptor. Thus, pro-caspase- 8 recruitment interacts with FADD through another domain, DED (death-effector domain), forming DISC (death-inducing signaling complex). This complex results in the activation and autocleavage of caspase- 8 and is subsequently internalized following the cascade of intracellular signaling (Muzio, et al., 1998; Kischkel, et al., 1995; AlgecirasSchimnich, et al., 2002; Barnhart, et al., 2003; Diemer, et al., 2009)

In particular, apoptosis modulation in SARS$\mathrm{CoV}$ infection seems to be directly associated with specific cell-type interactions, which can significantly influence the manifestations resulting from the pathology (Lal, 2010; Diemer, et al., 2009). At the respiratory tract, significant expression of ACE-2 has been described, a type 1 membrane protein that participates in the maturation of angiotensin, a peptide hormone that controls vasoconstriction and blood pressure. ACE-2 was expressed in epithelial cells, bronchi, bronchial sinuses, glands, alveolar monocytes and macrophages, triggering cell death and indicating probable viral routes based on cell susceptibility and viral infectivity. Therefore, it is noted the need for studies that promote debates about the modulation of apoptosis in animals and humans, infected by SARS-CoV (Ortiz-Prado, et al., 2020).

Severe Acute Respiratory Syndrome (SARS) originated in southern China in November 2002 and was taken to Hong Kong in February 2003. With spread in Asian countries, the disease has spread continuously. Risk factors include old age and chronic illnesses. It is transmitted by contaminated droplets or contaminated surfaces, with an incubation period of 6-4 days and a period for clinical manifestations of 3-5 days. SARS-related Coronavirus 2 (SARS-CoV-2), recognized for being the etiologic agent of Severe Acute Respiratory Syndrome (SARS) in humans, is a newly identified member of the Coronaviridae family. SARS-CoV is enveloped and shares a similar composition and structure with other coronaviruses, with a genome of approximately $29.7 \mathrm{~kb}$ in length and is organized with an open reading frame (ORF), replication coding, "spike" (S), envelope (E), membrane $(\mathrm{M})$ and nucleocapsid $(\mathrm{N})$, in the sequence of 5 ' to 3' terminals (Ren, et al., 2005).

Little is understood about the origins of the SARS virus and its possible role or impact on the host cell, with the need for studies aimed at the interposition of viruses of the same family, in order to promote the screening of the interaction mechanisms already known and described in the literature, to track and be able to stipulate predictions based on the likelihood between its main aspects and pathogenic functions (Chan-Yeung; $\mathrm{Xu}$, 2003).

Therefore, there is a need for studies that correlate apoptosis and cell death, as well as replication and cytopathic effects in mammalian cell culture systems infected with SARS. Since apoptosis potentiates viral propagation and conditions the inflammatory reaction in the host in viral infection (Ren, et al., 2005).

From this, it is possible to suggest that understanding about these events can provide knowledge based on the theoretical and experimental basis applied in the development of new antiviral strategies.

\section{Materials and Methods}

This study is characterized as a short communication, conducted from a narrative 
perspective, under an observational and transversal approach. The main method was the search for studies related to the activation of caspases in SARS-CoV infection. From the delimitation of the search criteria, there was a comparison between the selected articles and presentation of the obtained results.

The elaboration of the study was concerned with providing the outline of the execution of the research, with emphasis on the execution of the proposed theme, as well as its importance for the scientific community. In order to configure a specific screening in the search for research products, there was, in principle, the formulation of the guiding question. Based on this, the inclusion and exclusion criteria were subdivided, the definition of the search filters, construction of data collection instruments, consultation and selection of descriptors. After obtaining the study material, the approach to the construction of this work was structured, through the evaluation and analysis of selected articles, more relevant products, interpretation and discussion of the results obtained and presentation of the final article.

Regarding the period, the survey of the content was carried out from November 2020 to March 2021. The descriptors used were: SARS-CoV, Coronavirus, Covid, Caspases. As an eligibility criterion, original studies were considered that evaluated the activation of caspases in mammalian cells, from SARS $\mathrm{CoV}$ infection, in the period between 2011 and 2021, available with full text.

Therefore, in relation to the methodological consistencies of the selected studies, data were extracted based on information configured in the following variables: (i) the author, and year of publication of the article; (ii) experimental analysis studies; (iii) molecular techniques used and cell lines; (iv) mechanisms of cell interaction and viruses; (v) importance and contribution of the results presented in the study.

\section{Results and Discussion}

Because the spread of SARS-CoV-2 has become the most recent threat to global health, the quest to understand the virus-host interaction mechanisms has become a challenge for public health, research and medical communities, especially due to the interposition of factors related to the evolutionary spectrum of the agent. To this end, an alternative that became viable in tracking the development of antiviral strategies fundamentally based on multifactorial approaches associated with the variety of experimental studies with investigative approaches, was the description of the main effect or molecules responsible for the infection progression. as well as the identification of caspases subsequently activated as a defense mechanism (YAN, et al., 2004; BORDI, et al., 2006). This immune response, resulting from stimuli from the recognition of pathogenic agents, acts as an important barrier against coronavirus infection reaching the oral or nasal cavity, being directed to the respiratory epithelium and epithelial cells. MHV, SARS-CoV, MERS$\mathrm{CoV}$ and SARS-CoV-2 can infect immune and non-immune cells in the respiratory tract, being recognized by several standard recognition receptors. Thus, seeking to understand this cascade of processes involved during infection by viruses belonging to the same family can contribute to the understanding about the progression of Coronavirus Disease 2019 (COVID-19).

Ren, et al., (2020) analyzed the induction of apoptosis from SARS-CoV-2 ORF3a, in HEK293T, HepG2 and Vero E6 cell cultures in vitro infection. It was found that the virus induced caspase- 8 activation (cleavage), while the level of Bcl-2 expression was not altered. 
These indicators were already expected, since the activation of caspase- 8 is recognized as a trademark of the extrinsic apoptotic pathway, while on the other hand, Bcl-2 has a fundamental role in the initiation of the intrinsic pathway. It was also possible to identify high levels of truncated Bid (tBid), caspase-9 and cytochrome $\mathrm{c}$, in addition to significant suppression of apoptosis by an inhibitor of caspase- 8 and caspase- 9 , in the presence of SARS-CoV-2 ORF3a. Thus, the results obtained indicate a significant induction of apoptosis by the extrinsic pathway by SARS-CoV-2, associated with caspase-8, which in turn cleaves tBid and corroborates the release of mitochondrial cytochrome c, causing the formation of apoptosome and activation of caspase-9. A similar study investigated whether SARSCoV-2 infection induces caspase activation and subsequent cell apoptosis and inflammatory cytokine processing. It was also observed that activation of caspase- 8 and expression of IL-1 $\beta$ in the bioactive form secreted by virus through the necroptosis pathway induced inflammatory responses (LI, et al., 2020). The authors also reported the onset of inflammatory processes and cell death in lung cells through the activation of caspase8 , processing inflammatory factors such as IL$1 \beta$.

Among the selected studies, Tsoi et al., (2014) demonstrated that there is a $M$ protein expression in SARS-CoV infection, causing a negative regulation of phosphorylation and subsequent enrichment of FKHRL1 in the nuclear compartment - which is a responsible transcription factor for controlling the levels of FasL expression - resulting in the eventual activation of caspase-8. In addition, indicators presented by the authors allow us to assume that caspase- 8 is probably not the only mediator of cell death induced by protein $\mathrm{M}$, since, after caspase- 8 blockade, caspase- 9 activity only partially blocked cell death. In general, it was possible to establish that $\mathrm{M}$ protein induces activation of caspase- 8 and 9, in addition to negative PKB-Akt regulation of the signaling cascade, compromising cell survival, signaling of viral agents, which may later cause apoptosis.

Another study, carried out from infection by the same family viruses, described different results. Dimer, et al., (2007), demonstrated in his study, that SARS-CoV-1 can assume the role of a substrate active in caspase-mediated cleavage after viral infection or by transient transfection in different cell lines, mainly associated with protein $\mathrm{N}$. The authors also describe, by experimental techniques applied to immunocytochemical methods, that the cleavage of caspase is directly linked to the cell line, associated with the nuclear localization of protein $\mathrm{N}$ in SARS-CoV-1 infection (Vero, Caco-2). The protein $\mathrm{N}$ detected in Vero cells causes the inhibition of the activation of caspase- 1 and caspase-3, indicating that the inhibition of caspase-3 prevents the activation of caspases 6 and 7, responsible for being the executors of apoptosis and finalizing the cascade. In this perspective, the induction of apoptosis, related to $\mathrm{N}$ dependence by caspase- 6 , has its specificity based primarily on the cell line, correlated with the activation of survival pathways in persistently infected cells. In general, it was possible to establish that there is a probable relationship between the nuclear localization of proteins or specific genes that can act on the caspase activation, expressed from the lytic mechanisms or persistent SARS-CoV infection.

Many viral genomes that can encode gene products responsible for modulating apoptosis, are described as determinants associated with the regulation of antiviral defense mechanisms. In this perspective, Tan, et al., (2007) associate the modulation of cell death in SARS-CoV infection and the interaction of Bcl-XL dependent on Accessory Protein 7, with viral replication and pathogenesis. The 
study showed that there is a quantitatively high expression of the accessory protein SARS-CoV 7a, inducing apoptosis by caspase-3-dependent activation and that the induction of $7 \mathrm{a}$ apoptosis can be restricted based on the expression of Bcl-X, characterized as a member of the Bcl-2 family, and may be related to the amount of the Bcl-2 family in $7 \mathrm{a}$. These considerations may suggest the hypotheses related to the influence of mediator molecules expressed during infection, in the activation of caspases, since the described results reported that there were no significant changes in the replication of SARS-CoV from the inhibition of apoptosis, raising questions about the diversity of viral pathogenesis.

Similar results to those described in the literature, Ye, et al., (2008) reported expression of the SARS ORF-6 protein (fourth accessory protein) inducing activation of apoptosis due to caspase- 3 dependence. It was also identified, via c-Jun N-terminal kinase inhibitor (JNK), inducing apoptosis by ORF-6 and OFR-7, causing stress and impairment of the endoplasmic reticulum, suggesting the occurrence of alternative reactions, as well as inhibition glycolysis, reduction of disulfide bonds, calcium depletion, impaired protein transport to the golgi complex, and expression of several mutated proteins. Thus, although there is no homology between ORF-6 and known proteins, the authors were able to associate the molecular pathway for apoptosis, induced by ORF-6 similar to ORF-7a, curiously associated with the studies previously described. Based on this bias, a specific approach should be carried out, based on the parallelism of over expression of apoptosis inducing and inhibiting proteins to track their influence on human cells infection. The importance of characterizing the profile of the immune response triggered by different $\mathrm{CoV}$ infections converges in apoptosis similarities associated with viral pathogenesis and innate immune response. Thus, given the urgency of the global health situation, the need to develop therapies and vaccines to combat Covid-19 due to the SARS-CoV-2 pandemic that the recent experimental data on the main molecules expressed during infection by SARS-CoV-2, can be an alternative for the development of new effective parameters in the control of the disease, is the recognition and the differential parallelism between the mediating molecules, by which the immune system recognizes and responds to specific $\mathrm{CoV}$ infections, acting to support the development of therapeutic strategies.

\section{References}

Algeciras-Schimnich A, Shen L, Barnhart BC, Murmann AE, Burkhardt JK, PETER ME. Molecular ordering of the initial signaling events of CD95. Mol Cell Biol. v. 22, e. 1, p. 207-20 2002.

Barnhart BC, Lee JC, Alappat EC, Peter ME. The death effector domain protein family. Oncogene. v. 22, e. 53, p. 8634-44, 2003.

Bordi, L., C. Castilletti, L. Falasca, F. Ciccosanti, S. Calcaterra, G. Rozera, A. DI Caro, S. Zaniratti, A. RINALDI, G. Ippolito, M. Piacentini, and M. R. Capobianchi. 2006. Bcl-2 inhibits the caspase-dependent apoptosis induced by SARS-CoV without affecting virus replication kinetics. Arch. Virol. 151:369-377

Chan-Yeung, M.; Xu, R. SARS : epidemiology Cumulative NUMBER of cases and Deaths in various countries In. Respirology, v. 8, p. S9-S14, 2003.

Diemer C.; Schneider M.; Schatzi, H. M.; Hermann, MoM. H.; Glich, S. Modulation of Hort Cell Death by SARS Coronavirus Proteins. Nature Public Health Emergency Collection, v. 22, p. 231-245, 2009.

Diemer, C. et al., Cell Type-Specific Cleavage of Nucleocapsid Protein by Effector Caspases during SARS Coronavirus Infection. Journal of Molecular Biology, v. 376, n. 1, p. 23-34, 2008.

Kischkel FC, Hellbardt S, Behrmann I, Germer M, Pawlita M, Krammer PH, et al., Cytotoxicity-dependent $\quad$ APO-1 
(Fas/CD95)-associated proteins form a death- inducing signaling complex (DISC) with the receptor. The EMBO Journal. v. 15 , n. 22, p. 5579-88, 1995.

Kurosaka, K. et al., Silent Cleanup of Very Early Apoptotic Cells by Macrophages. The Journal of Immunology, v. 171, n. 9, p. 4672-4679, 2003.

Lal, S. K. Molecular biology of the SARScoronavirus. Molecular Biology of the SARS-Coronavirus, p. 1-328, 2010.

Lavrik, I. N.; Golks, A.; Krammer, P. H. Caspase: Pharmacological manipulation of cell death. Journal of Clinical Investigation, v. 115, n. 10, p. 2665-2672, 2005.

LI, S. et al., SARS-CoV-2 triggers inflammatory responses and cell death through caspase-8 activation. Signal Transduction and Targeted Therapy, v. 5, n. 1, 2020.

Muzio M, Stockwell BR, Stennicke HR, Salvesen GS, Dixit VM. An induced proximity model for caspase-8 activation. The Journal of Biological Chemistry. v. 273, n. 30 p. 2926-30, 1998.

Ren, Y. et al., The ORF3a protein of SARS-CoV-2 induces apoptosis in cells. n. May, p. 810, 2020.

Ren, L. et al., Apoptosis induced by the SARSassociated coronavirus in vero cells is replication-dependent and involves caspase. DNA and Cell Biology, v. 24, n. 8, p. 496-502, 2005.

Obeng, E. Apoptosis (programmed cell death) and its signals - A review. Brazilian Journal of Biology, v. 81, n. 4, p. 1133-1143, 2021.

Ortiz-Prado, E. et al., Clinical, molecular, and epidemiological characterization of the SARS-CoV-2 virus and the Coronavirus Disease 2019 (COVID-19), a comprehensive literature review. Diagnostic Microbiology and Infectious Disease, v. 98, n. 1, p. 115094, 2020.

Savill, J.; Fadok, V. Corpse clearance defines the meaning of cell death. Nature, v. 407, n. 6805, p. 784-788, 2000.

Tan, Y.-X. et al., Induction of Apoptosis by the Severe Acute Respiratory Syndrome Coronavirus 7a Protein Is Dependent on Its Interaction with the Bcl-XL Protein. Journal of Virology, v. 81, n. 12, p. 63466355, 2007.

Tsoi, H. et al., The SARS-coronavirus membrane protein induces apoptosis via interfering with PDK1PKB/Akt signalling. Biochemical Journal, v. 464, n. 3, p. 439447, 2014.

Yan, H., G. Xiao, J. Zhang, Y. Hu, F. Yuan, D. K. Cole, C. Zheng, and G. F. Gao. 2004. SARS coronavirus induces apoptosis in Vero E6 cells. J. Med. Virol.73:323-331

Ye, Z. et al., A SARS-CoV protein, ORF-6, induces caspase-3 mediated, ER stress and JNK-dependent apoptosis. Biochimica et Biophysica Acta - General Subjects, v. 1780, n. 12, p. 1383-1387, 2008.

Zeiss, C. J. The apoptosis-necrosis continuum: Insights from genetically altered mice. Veterinary Pathology, v. 40, n. 5, p. 481495, 2003.

Ziegler, U.; Groscurth, P. Morphological features of cell death. News in Physiological Sciences, v. 19, n. 3, p. 124-128, 2004.

Zhang L.; Wei L.; Jiang D.; Wang J.; Cong X.; Fei R. SARS-CoV nucleocapsid protein induced apoptosis of COS-1 mediated by the mitochondrial pathway. Artif Cells Blood Substit Immobil Biotechnol. v. 35, p. 237-253. 2007.

\section{How to cite this article:}

Jardel Fábio Lopes Ferreira, Murilo Tavares Amorim, Francisco Canindé Ferreira de Luna, Gustavo Moraes Holanda, Clebson Pantoja Pimentel, Walter Félix Franco Neto, Caroline Carvalho Pinto, Ana Paula Sousa Araújo, Gleissy Adriane Lima Borges and Ana Cecília Ribeiro Cruz. 2021. SARS-CoV-2 Triggers Inflammatory and Cell Death in Experimental Infection by Activating Human Cells Caspases. Int.J.Curr.Microbiol.App.Sci. 10(04): 853-859. doi: https://doi.org/10.20546/ijcmas.2021.1004.089 